\title{
ALGEBRAIC DETERMINATION OF FIBERWISE PL INVOLUTIONS
}

BY

\author{
HAYON KIM, JEHPILL KIM AND KYUNG WHAN KWUN ${ }^{1}$
}

\begin{abstract}
Some fiberwise PL involutions on fibered 3-manifolds induce the obvious automorphism of the fundamental group. It is shown that this expected behavior of the fundamental group in turn characterizes such fiberwise involutions.
\end{abstract}

1. Introduction. Much information about a PL involution $h$ of a compact 3-manifold $X$ can be obtained from analysing the isomorphism $h_{\sharp}: \pi_{1}(X) \rightarrow \pi_{1}(X)$. See, for example, [13], [5]. For particularly striking results in case $\pi_{1} X$ is centerless, see [13]. This paper characterizes certain PL involutions by $h_{\sharp}$.

A fibering of a group $G$ (over $\mathbf{Z}$ ) consists of a finitely generated normal subgroup $K$, the fiber, and an infinite cyclic subgroup $H$, a cross-section, such that $G=K H$ and $K \cap H=1$. It is denoted by $\{K, H\}$. By a transversal element of the fibering, we mean an element not in the fiber. An involution $\sigma$ of $G$ is fiberwise if for some fibering $\{K, H\}, \sigma(K)=K$ and $\sigma$ fixes a transversal element. An involution $\sigma$ of $G$ is fiberwise with invariant cross-section if for some $\{K, H\}, \sigma(K)=K$ and $\sigma \mid H=$ $\mathbf{l}_{H}$.

A PL involution $h$ of a compact 3-manifold $X$ is said to be fiberwise (or fiberwise with invariant cross-section) if there is a fibering $g: X \rightarrow S^{1}$ such that each fiber is invariant under $h$ (or each fiber is invariant under $h$ and a subset of the fixed point set is mapped homeomorphically onto $S^{1}$ under $g$ ). Fibers are assumed to be connected.

THEOREM. Let $h$ be a PL involution with fixed point set $F \neq \varnothing$ of a closed $P^{2}$-irreducible 3-manifold $X$. Then

(a) $h$ is fiberwise if and only if $h_{\sharp}: \pi_{1}\left(X, x_{0}\right) \rightarrow \pi_{1}\left(X, x_{0}\right)$ is a fiberwise involution for some fixed point $x_{0}$,

(b) $h$ is a fiberwise involution with invariant cross-section if and only if $h_{\sharp}$ is a fiberwise involution with invariant cross-section of $\pi_{1}\left(X, x_{0}\right)$ for some fixed point $x_{0}$ of $h$.

COROllaRY. Let $M$ be an orientable closed surface not homeomorphic to $S^{2}$. Let $h$ be a PL involution of $M \times S^{1}$ with one-dimensional fixed point set $F$. Then $h$ is equivalent to $\alpha \times 1_{S^{1}}$ for some involution $\alpha$ of $M$ if and only if under some product

\footnotetext{
Received by the editors February 22, 1980 and, in revised form, September 3, 1980.

AMS (MOS) subject classifications (1970). Primary 57A10.

Key words and phrases. PL involutions, fibering, fiberwise, fibered 3-manifolds.

1 The third author is supported in part by NSF Grant MCS 76-07301.
}

(C) 1981 American Mathematical Society 0002-9947/81/0000-0408/\$02.75 
structure of $M \times S^{1}$ and $\left(m_{0}, s_{0}\right) \in F$, the automorphism $h_{\sharp}$ of $\pi_{1}\left(X,\left(m_{0}, s_{0}\right)\right)=$ $\pi_{1}\left(M, m_{0}\right) \times \pi_{1}\left(S^{1}, s_{0}\right)$ satisfies conditions

$$
h_{\sharp}\left(\pi_{1}\left(M, m_{0}\right)\right)=\pi_{1}\left(M, m_{0}\right)
$$

and $h_{\sharp} \mid \pi_{1}\left(S^{1}, s_{0}\right)$ is the identity.

The "only if" parts of the theorem for (a) and (b) are obvious. Proofs for the "if" parts of (a) and (b) are virtually the same. From here on we will exclusively discuss part (b). After the proof of part (b), we will indicate how to prove part (a) and the Corollary. Henceforth, we assume $h_{\sharp}$ is fiberwise with invariant cross-section and will prove that $h$ is fiberwise with invariant cross-section. Let $X, F, h, x_{0}$ be as in the theorem(b).

2. The covering involution $\tilde{h}$. Let $\{K, H\}$ be a fibering of $\pi_{1}\left(X, x_{0}\right)$ making $h_{\#}$ fiberwise with invariant cross-section. Let $q:\left(\tilde{X}, \tilde{x}_{0}\right) \rightarrow\left(x, x_{0}\right)$ be the covering projection corresponding to the subgroup $K$. It can be shown that $K \nsim \mathbf{Z}_{2}$ under our assumption. The fibering theorem [10] of Stallings then implies that $\tilde{X}$ is homeomorphic to $M \times \mathbf{R}^{1}$ for some closed surface $M$ with $\pi_{1} M \simeq K$.

By [3, Theorem 6.1, p. 245], the component $F_{0}$ of $F$ containing $x_{0}$ contains a loop representing a generator $t$ of $H$. Since $K H / K \simeq H /(K \cap H), K \cap H=1, H$ may be viewed as the group of covering transformations of $q$. Hence $\tilde{F}_{0}=q^{-1}\left(F_{0}\right)$ is connected. Let $T$ generate the group of covering transformations of $q$.

LemMA 2.1. Let $\tilde{h}$ be the involution of $\left(\tilde{X}, \tilde{x}_{0}\right)$ such that $q \tilde{h}=h q$. Then $\tilde{h} T=T \tilde{h}$.

Proof. Such $\tilde{h}$ exists as $h_{\sharp}(K)=K$. Also $\tilde{h}$ fixes $\tilde{F}_{0}$ pointwise. Clearly $T\left(\tilde{F}_{0}\right)=$ $\tilde{F}_{0} \cdot \tilde{h} T\left(\tilde{x}_{0}\right)=T\left(\tilde{x}_{0}\right)=T \tilde{h}\left(x_{0}\right)$. Also since $q \tilde{h} T=h q T=h q=q \tilde{h}=q T \tilde{h}$, it follows that $\tilde{h} T=T \tilde{h}$.

LeMMA 2.2. Let $\tilde{F}$ be the fixed point set of $\tilde{h}$. Then $\tilde{F}=q^{-1}(F)$.

Proof. Certainly $\tilde{F} \subset q^{-1}(F)$. Let $\tilde{x}_{1} \in q^{-1}(F)$ and $x_{1}=q\left(\tilde{x}_{1}\right)$. Let $\gamma$ be a path from $x_{1}$ to $x_{0}$. With respect to the new base point $x_{1}$, the covering projection $q$ corresponds to $K^{\prime} \subset \pi_{1}\left(X, x_{1}\right)$ obtained from $K$ and $\gamma$. It can be checked that $h_{\sharp}\left(K^{\prime}\right)=K^{\prime}$. (In general, it does not follow that $h_{\sharp} \mid H^{\prime}$ is the identity, where $H^{\prime}$ is obtained from $H$ and $\gamma$.) Hence there exists an involution $\tilde{h}^{\prime}$ of $\left(\tilde{X}, \tilde{x}_{1}\right)$ such that $q \tilde{h}^{\prime}=h q$. Then $\tilde{h} \tilde{h}^{\prime}$ is a covering transformation. If $\tilde{h} \tilde{h}^{\prime}=T^{i}$ for some integer $i$, $\tilde{h}^{\prime}=\tilde{h} T^{i}$ and $1_{\tilde{X}}=\tilde{h}^{\prime} \tilde{h}^{\prime}=T^{2 i}$. Hence $i=0$ and $\tilde{h}^{\prime}=\tilde{h}$. Hence $\tilde{h}$ fixes $\tilde{x}_{1}$.

Lemma 2.3. Regarding $\tilde{X}$ as $M \times \mathbf{R}^{1}, \tilde{h}$ is equivalent to $q \times 1_{\mathbf{R}^{1}}$, where $\alpha$ is an involution of $M$.

Proof. According to the extension theorem [6], $\tilde{h}$ is equivalent to the restriction to the interior of a PL involution of $M \times[0,1]$. The conclusion now follows from the product theorem [4] of Kim and Tollefson and the fact that $\tilde{F}$ is not compact.

RemarK. Since $\tilde{F}$ is a disjoint union of lines and open annuli, $F$ is a disjoint union of simple closed curves, tori and Klein bottles. There is no 0-dimensional component of $F$. Also notice that even though $q^{-1}\left(F_{0}\right)$ is connected, we did not show that the same is true for other components of $F$. Simple examples show that this is not the case in general. 
Let $f: \tilde{X} \rightarrow \tilde{Y}$ and $f_{0}: X \rightarrow Y$ be orbit maps of $\mathbf{Z}_{2}$ actions generated by $\tilde{h}$ and $h$, respectively. Let $\beta: M \rightarrow N$ be the orbit map of the $Z_{2}$ action generated by $\alpha$.

LEMMA 2.4. $\tilde{Y}$ is homeomorphic to $N \times \mathbf{R}^{1}$ and there is a commutative diagram

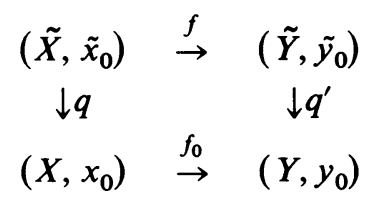

where $q^{\prime}$ is a covering projection whose group of covering transformations is generated by $T^{\prime}, T^{\prime}$ being induced by $T$.

Proof. Everything is either obvious or follows from the commutativity $T \tilde{h}=h \tilde{T}$.

3. The orbit space $Y$ of $h$.

LEMMA 3.1. $Y$ is fibered over $S^{1}$ with fiber $S$ homeomorphic to $N$ and $\pi_{1}(S)=$ $q_{\sharp}^{\prime} \pi_{1}(\tilde{Y})$.

Proof. First observe that $Y$ contains no fake 3-cell as its universal covering space embeds in $\mathbf{R}^{3}$.

Case 1. $N \not S^{2}$ and $N \not P^{2}$. In this case, $Y$ is irreducible as $N$ is aspherical. Hence the result follows from Stallings [10].

Case 2. $N \approx S^{2}$ or $N \approx P^{2}$. Since $\dot{Y}=\varnothing$ and $\pi_{1}(Y) \approx \mathbf{Z}$ or $\mathbf{Z}+\mathbf{Z}_{2}$ is not a free product, $Y$ must be prime. If $Y$ is orientable, since $\pi_{2}(Y) \approx \mathrm{Z}$, the sphere theorem [9] implies that $Y$ cannot be irreducible. Hence $Y \approx S^{2} \times S^{1}$ by [8]. If $Y$ is not orientable, let $Y^{\prime}$ be the orientable double covering space of $Y$. Since $Y^{\prime}$ must be covered by the universal covering space $S^{2} \times \mathbf{R}^{1}$, no fake 3-cell exists in $Y^{\prime}$. Hence the argument in the orientable case shows that $Y^{\prime}=S^{2} \times S^{1}$. Therefore, by [12], either $Y$ is the nonorientable $S^{2}$-bundle over $S^{1}$ or $Y \approx P^{2} \times S^{1}$ according as $N \approx S^{2}$ or $N \approx P^{2}$. Observe that $S$ is in fact homemorphic to $N$ as

$$
N \times \mathbf{R}^{1} \approx \tilde{Y} \approx S \times \mathbf{R}^{1} .
$$

A curve and a surface in general position will be said to have the intersection number \pm 1 if the curve crosses the surface once algebraically. We will denote $f_{0}\left(F_{0}\right)$ by $F_{0}^{\prime}$.

LEMMA 3.2. The fibering of Lemma 3.1 may be chosen so that the fiber $S$ containing $y_{0}$ and $F^{\prime}$ is in general position. If $F_{0}^{\prime}$ is 1-dimensional, the intersection number of $F_{0}^{\prime}$ and $S$ is \pm 1 and if $F_{0}^{\prime}$ is 2-dimensional $F_{0}^{\prime} \cap S$ is a simple closed curve whose complement in $F_{0}^{\prime}$ is an open annulus.

Proof. The first part is straightforward. For the second part, consider the commutative diagram with exact rows

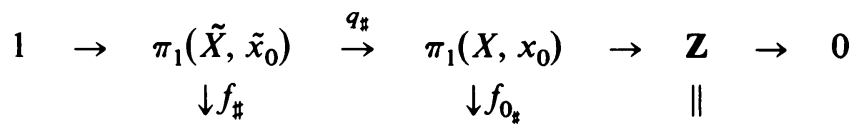

$$
\begin{aligned}
& 1 \rightarrow \pi_{1}\left(\tilde{Y}, \tilde{y}_{0}\right) \stackrel{q_{\pi}^{\prime}}{\rightarrow} \pi_{1}\left(Y, y_{0}\right) \rightarrow \mathbf{Z} \rightarrow 0
\end{aligned}
$$


where Z's are generated by $T$ and $T^{\prime}$. Since the composite $\pi_{1}\left(F_{0}, x_{0}\right) \rightarrow \pi_{1}\left(X, x_{0}\right) \rightarrow$ $\mathbf{Z}$ is epimorphic, the lemma will follow if we can identify the bottom row with the exact homotopy sequence of the fibering.

$$
1 \rightarrow \pi_{1}\left(S, y_{0}\right) \rightarrow \pi_{1}\left(Y, y_{0}\right) \rightarrow \pi_{1} S^{1} \rightarrow 0 .
$$

If $N \not z S^{2}$ or $P^{2}$, this follows from the way Stallings proves the fibering theorem. If $N \approx S^{2}$ or $P^{2}$, this is automatic since the kernel of any epimorphism $\pi_{1}\left(Y, y_{0}\right) \rightarrow \mathbf{Z}$ is unique.

LEMMA 3.3. In Lemma 3.2, it may be further assumed that $S-F^{\prime} \subset Y-F^{\prime}$ induces a monomorphism of $\pi_{1}$.

Proof. If the conclusion does not hold already, by the loop theorem [11], there exists a disk $D \subset Y-F^{\prime}$ such that $D \cap\left(S-F^{\prime}\right)=\dot{D}$, the boundary of $D$, is not deformable to a point in $S-F^{\prime}$.

Case 1. $S \approx S^{2}$. Since $S$ is incompressible $\dot{D}$ bounds a disk $D^{\prime}$ in $S$. Clearly $D^{\prime} \cap F^{\prime} \neq \varnothing$. Since $Y$ is irreducible $D$ and $D^{\prime}$ together bound a 3-cell and there is an isotopy of $S$ to $S^{\prime}=\left(S-D^{\prime}\right) \cup D$. Now $S^{\prime}$ may be considered as the new fiber. $S^{\prime}$ intersects $F^{\prime}$ at fewer points. Repeat this process. This process must end as $S^{\circ}$ intersects $F^{\prime}$ at a finite number of points.

Case 2. $S \approx S^{2} . \dot{D}$ separates $S$ into two disks $D_{1}$ and $D_{2} . D_{1} \cap F^{\prime} \neq \varnothing \neq D_{2} \cap$ $F^{\prime}$. Using mod 2 homology, since $S$ does not bound anything, exactly one of $D_{1} \cup D$ and $D_{2} \cup D$ bounds a 3-cell. If $D_{1} \cup D$ bounds a 3-cell, let $S^{\prime}=(S-$ $\left.D_{1}\right) \cup D$. The rest of the argument is the same as before.

4. The orbit space $\tilde{Y}$ of $\tilde{h}$. Choose a component $N_{0}$ of $q^{-1}(S)$, where $S$ is as in Lemma 3.3. Let $N_{i}=T^{\prime i}\left(N_{0}\right)$, where $i=0, \pm 1, \pm 2, \ldots$ Each $N_{i}$ maps homeomorphically onto $S$ under $q^{\prime}$. Let $W_{i}$ be the compact 3-manifold bounded by $N_{i}$ and $N_{i+1}$.

LEMMA 4.1. For each $i, N_{i}-\tilde{F}^{\prime} \subset W_{i}-\tilde{F}^{\prime}$ and $N_{i+1}-\tilde{F}^{\prime} \subset W_{i}-\tilde{F}^{\prime}$ induce isomorphisms of $\pi_{1}$, where $\tilde{F}^{\prime}=f(\tilde{F})$.

Proof. Both induce monomorphisms by Lemma 3.3. If either fails to induce an epimorphism, it follows that $\pi_{1}\left(\tilde{Y}-\tilde{F}^{\prime}\right)$ is not finitely generated. But $\tilde{Y}-\tilde{F}^{\prime}$ is the same homotopy type as $N-\beta$ (fixed point set of $\alpha$ ), where $\beta: M \rightarrow N$ is the orbit map of $\alpha$. (See the part preceding Lemma 2.4.)

LEMMA 4.2. The interior $\stackrel{\circ}{*}_{i}$ of each $N_{i}$ intersects each 1-dimensional component of $\tilde{F}^{\prime}$ exactly at a single point.

Proof. Let $B$ be the finite subset of $N$ corresponding to isolated fixed points of $\alpha: M \rightarrow M$ under the orbit map $\beta$. Then $\tilde{Y}-\tilde{F}^{\prime}$ is homeomorphic to $(\stackrel{\circ}{N}-B) \times$ $\mathbf{R}^{1}$. Now by Lemma 4.1, $\pi_{1}(\stackrel{\circ}{N}-B) \approx \pi_{1}\left(\tilde{Y}-\tilde{F}^{\prime}\right) \approx \pi_{1}\left(\stackrel{\circ}{N}_{i}-\tilde{F}^{\prime}\right)$. Hence the cardinality of $\stackrel{\circ}{N}_{i} \cap \tilde{F}^{\prime}$ is exactly the number of 1-dimensional components of $\tilde{F}^{\prime}$. Since each component of $\tilde{F}^{\prime}$ intersects $N_{i}$, the lemma follows. 


\section{The covering space $\tilde{X}$.}

LemMA 5.1. Let $f^{-1}\left(N_{i}\right)=M_{i}$. Then $M_{i}$ separates $\tilde{X}$ and $M_{i} \approx M$.

Proof. The first part is obvious. For the second part, observe that $\dot{N}_{i} \subset \tilde{F}^{\prime}$. This together with Lemma 4.2 implies $M$ and $M_{i}$ have the same Euler characteristic. Furthermore $M_{i}$ is a closed surface. The both are two sided in $\tilde{X}$; their orientabilities coincide. ${ }^{2}$ The lemma now follows.

LeMmA 5.2. ${ }^{3}$ Let $K$ be a closed surface and let $K^{\prime} \approx K, K^{\prime} \subset K \times(0,1)$. Suppose $K^{\prime}$ separates $K \times 0$ from $K \times 1$. Then $K^{\prime} \subset K \times[0,1]$ is a homotopy equivalence.

Proof. This must be well known. Here is a proof. First suppose $K$ is orientable. $K \times 0$ and $K^{\prime}$ together bound an orientable compact 3-manifold. Hence they are homologous. Hence $i_{*}: H_{2}\left(K^{\prime}\right) \approx H_{2}(K \times[0,1])$ over $\mathrm{Z}$, where $i: K^{\prime} \subset K \times[0,1]$. Let $p: K \times[0,1] \rightarrow K$ be the projection. Then $p i: K^{\prime} \rightarrow K$ is of degree \pm 1 . Hence $(p i)_{\sharp}: \pi_{1}\left(K^{\prime}\right) \rightarrow \pi_{1}(K)$ is an epimorphism. Since $\pi_{1}\left(K^{\prime}\right) \approx \pi_{1}(K)$ is hopfian, $(p i)_{\sharp}$, therefore $i_{\sharp}$ also, is an isomorphism. If $K$ is not orientable, let $\tilde{K}$ be the orientable double covering space. Let $\tilde{K} \times[0,1] \stackrel{p^{\prime}}{\rightarrow} K \times[0,1]$ be the double covering and $\tilde{K}^{\prime}=p^{\prime-1}\left(K^{\prime}\right) . \tilde{K}^{\prime}$ is connected as $K^{\prime}$ is 2-sided and nonorientable. Since $\tilde{K}^{\prime}$ separates $\tilde{K} \times[0,1], \tilde{K}^{\prime} \subset \tilde{K} \times[0,1]$ is a homotopy equivalence. Then it follows that if $\pi_{1}\left(K^{\prime}\right) \rightarrow \pi_{1}(K \times[0,1])$ is not an epimorphism; the kernel is $\mathbf{Z}_{2}$. This is possible only if $K \approx P_{2}$. But if this happened for $K \approx P^{2}$, one would be able to embed $P^{2}$ in $S^{2} \times[0,1]$. This is impossible.

6. Proof of Theorem and Corollary. Let $f^{-1}\left(W_{i}\right)=U_{i}$, where $W_{i}$ are as in $\$ 4$. By Lemma 5.2 and the finite generation of $\pi_{1} \tilde{X}, M_{0} \subset U_{0}$ and $M_{1} \subset U_{0}$ can be seen to be homotopy equivalences. Since there is no fake 3-cell in $\tilde{X} \approx M \times \mathbf{R}^{1},\left(U_{0}, M_{0}\right)$ is homeomorphic to $M \times([0,1],\{0\})$ by [2]. Clearly $M_{1}=T\left(M_{0}\right)$ and $\tilde{h}\left(U_{0}\right)=U_{0}$. By the product theorem of $\mathrm{Kim}$ and Tollefson, $\tilde{h} \mid U_{0}$ is equivalent to $\alpha^{\prime} \times 1$ of $M \times[0,1]$ where $\alpha^{\prime}$ is an involution of $M$. Since $h$ and $X$ are obtained from $\tilde{h} \mid U_{0}$ and $U_{0}$ by identifying $M_{0}$ and $M_{1}$ by the equivariant homeomorphism $T, h$ is fiberwise. An invariant cross-section can be easily found in $F_{0}$. Such cross-section represents an element $x t$, where $x \in K$ and $t$ generates $H$. This follows from Lemma 3.2 and is crucial in the proof of the Corollary.

To prove part (a), we first prove Lemma 2.1 in this case as follows. Because $h_{\sharp}$ fixes a transversal element, $q^{-1}\left(F_{0}\right)$ is the disjoint union of noncompact components. Choose $\tilde{x}_{1} \in q^{-1}\left(x_{0}\right) \cap \tilde{F}_{0}, \tilde{F}_{0}$ being the component of $q^{-1}(F)$ containing $\tilde{x}_{0}$ and $\tilde{x}_{1} \neq \tilde{x}_{0}$. There exists a nonzero integer $i$ such that $T^{i}\left(\tilde{x}_{0}\right)=\tilde{x}_{1}$. Since $\tilde{F}_{0}$ is pointwise fixed under $\tilde{h}, T^{i}$ commutes with $\tilde{h}$. Since $\tilde{h} T \tilde{h}$ is a covering transformation, $\tilde{h} T \tilde{h}=T^{j}$ for some $j$. Then $T^{i}=\tilde{h} T^{i} \tilde{h}=T^{i j}$. Since $i \neq 0, j=1$.

Lemma 3.2 is not true but it is not needed. The "monomorphism" part of Lemma 3.3 is still true and all that is needed. The rest of the argument is the same.

2 Added in proof. True but not completely obvious.

${ }^{3}$ Added in proof. Lemmas 5.1 and 5.2 are not needed. Use [2, Theorem 7.2, p. 504]. We regret the duplication. 
We prove the Corollary in one direction only. Let $K=\pi_{1}\left(M, m_{0}\right)$ and $H=$ $\pi_{1}\left(S^{1}, s_{0}\right)$. We recall that $X$ and $h$ are obtained from $\alpha^{\prime} \times 1$ and $M \times[0,1]$ by an equivariant homeomorphism $\phi$ of $M \rightarrow M \times 0 \rightarrow M \times 1 \rightarrow M$. By the above remark about the invariant cross-section and the fact $H$ is now central, it follows that $\phi$ is isotopic to the identity. The Corollary now follows from [1] if $M \approx S^{1} \times$ $S^{1}$. If $M \approx S^{1} \times S^{1}$, we simply check against [7].

7. Remarks. The proof of the Theorem actually implies that the fiberwise involution one finally obtains has a fiber corresponding to the $K$ one starts with.

In the following, we give an elementary construction which shows that the Corollary is false for any orientable closed surface of genus $>1$ if we simply assume that $h_{\sharp} \mid \pi_{1}\left(S^{1}, s_{0}\right)=$ identity. Let $M$ be the orientable surface of genus 2 and $\varphi$ be the involution of $M$ with exactly 6 fixed points. $M$ is pictured in Figure 1.

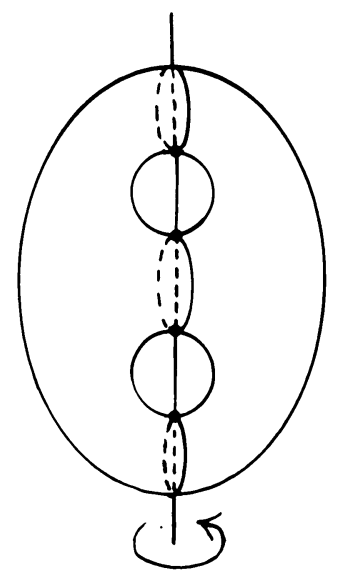

FIGURE 1

We define a map $M \stackrel{\psi}{\rightarrow} S^{1}$ as follows. The upper half (the part one sees in the picture) is pictured in Figure 2 and values at various points are shown. Here $S^{1}=\{$ all unit complex numbers $\}$.

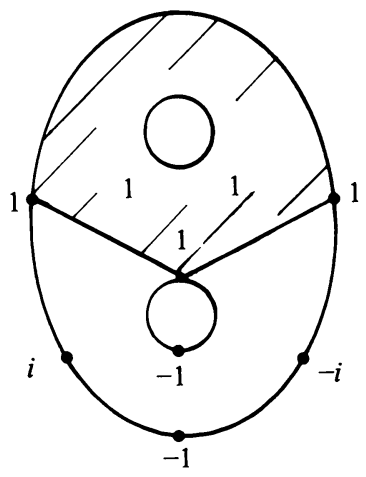

FigURE 2 
For $y$ in the lower half (the side we do not see) define $\psi(y)=(\psi(\varphi(y)))^{-1}$. The circle in the bottom of the picture goes around $S^{1}$ once. Define $h: M \times S^{1} \rightarrow M$ $\times S^{1}$ by

$$
h(x, t)=(\varphi(x), \psi(x) t) .
$$

Then $h$ is an involution equivalent to a PL involution. The fixed point set is equal to

$$
\{(x, t) \mid \varphi(x)=x, \psi(x)=1, t \text { arbitrary }\} .
$$

Therefore, it is the union of two disjoint simple closed curves each of which represents a generator of $\pi_{1}\left(S^{1}\right)$. However, $h$ is not equivalent to the product involution, because $\pi_{1}\left(S^{1}\right)$ is the center of $\pi_{1}\left(M \times S^{1}\right)$ and there is no involution of $M$ with two fixed points. The same construction works if genus $>2$.

\section{REFERENCES}

1. Joan S. Birman and Hugh M. Hilden, On isotopies of homeomorphisms of Riemann surfaces, Ann. of Math. (2) 97 (1973), 424-439.

2. E. M. Brown, Unknotting in $M^{2} \times I$, Trans. Amer. Math. Soc. 123 (1966), 480-505. MR 33 \#6640.

3. P. E. Conner and F. Raymond, Actions of compact Lie groups on aspherical manifolds, Topology of Manifolds (Proc. Inst., Univ. Georgia, 1969), Markham, Chicago, 1970, pp. 227-264. MR 42 \#6839.

4. P. K. Kim and J. L. Tollefson, PL involutions of fibered 3-manifolds, Trans. Amer. Math. Soc. 232 (1977), 221-237.

5. K. W. Kwun and F. Raymond, Periodic maps on product 3-manifolds which are isotopic to the identity, J. Korean Math. Soc. 16 (1980), 113-116.

6. K. W. Kwun and J. L. Tollefson, Extending a PL involution of the interior of a compact manifold, Amer. J. Math. 99 (1977), 995-1001.

7. $P L$ involutions of $S^{1} \times S^{1} \times S^{1}$, Trans. Amer. Math. Soc. 203 (1975), 97-106.

8. John Milnor, $A$ unique factorization theorem for 3-manifolds, Amer. J. Math. 84 (1962), 1-7.

9. C. D. Papakyriakopoulos, On Dehn's lemma and the asphericity of knots, Ann. of Math. (2) 66 (1957), 1-26. MR 19, 761.

10. John Stallings, On fibering certain 3-manifolds, Topology of 3-Manifolds and Related Topics, Prentice-Hall, Englewood Cliffs, N. J., 1961.

11. On the loop theorem, Ann. of Math. (2) 72 (1960), 12-19. MR 22 \#2526.

12. Yoko Tao, On fixed point free involutions of $S^{1} \times S^{2}$, Osaka J. Math. 14 (1962), 145-152.

13. J. L. Tollefson, Involutions of sufficiently large 3-manifolds (to appear).

Department of Mathematics, Concordia University, Montreal, Quebec, Canada

Department of Mathematics, Seoul National University, Seoul, Korea

Department of Mathematics, Michigan State University, East Lansing, Michigan 48824 